CUBO A Mathematical Journal Vol.20, $N^{\underline{O}_{3}}$, (49-63). October 2018

\title{
Study of global asymptotic stability in nonlinear neutral dynamic equations on time scales
}

\author{
Abdelouaheb Ardjouni ${ }^{1,2}$ And AhCene Djoudi ${ }^{2}$ \\ ${ }^{1}$ Department of Mathematics and Informatics, \\ University of Souk Ahras, \\ P.O. Box 1553, Souk Ahras, 41000, Algeria. \\ ${ }^{2}$ Applied Mathematics Lab, Faculty of Sciences, Department of Mathematics, \\ University of Annaba, \\ P.O. Box 12, Annaba 23000, Algeria. \\ abd_ardjouni@yahoo.fr, adjoudi@yahoo.com
}

\begin{abstract}
This paper is mainly concerned the global asymptotic stability of the zero solution of a class of nonlinear neutral dynamic equations in $\mathrm{C}_{\mathrm{rd}}^{1}$. By converting the nonlinear neutral dynamic equation into an equivalent integral equation, our main results are obtained via the Banach contraction mapping principle. The results obtained here extend the work of Yazgan, Tunc and Atan [17.
\end{abstract}

\section{RESUMEN}

Este artículo está mayormente interesado en la estabilidad global asintótica de la solución cero de una clase de ecuaciones nolineales neutrales dinámicas en $\mathrm{C}_{\mathrm{rd}}^{1}$. Transformando la ecuación nolineal neutral dinámica en una ecuación integral equivalente, nuestros resultados principales son obtenidos a través del principio de la aplicación contractiva de Banach. Los resultados obtenidos aquí son una extensión del trabajo de Yazgan, Tunc y Atan [17.

Keywords and Phrases: Fixed points, neutral dynamic equations, asymptotic stability, time scales.

2010 AMS Mathematics Subject Classification: 34K20, 34K30, 34K40. 
The concept of time scales analysis is a fairly new idea. In 1988, it was introduced by the German mathematician Stefan Hilger in his Ph.D. thesis [13. It combines the traditional areas of continuous and discrete analysis into one theory. After the publication of two textbooks in this area by Bohner and Peterson [7] and [8, more and more researchers were getting involved in this fast-growing field of mathematics. The study of dynamic equations brings together the traditional research areas of differential and difference equations. It allows one to handle these two research areas at the same time, hence shedding light on the reasons for their seeming discrepancies. In fact, many new results for the continuous and discrete cases have been obtained by studying the more general time scales case (see [1, 3, 4, 6, 14, and the references therein).

There is no doubt that the Lyapunov method have been used successfully to investigate stability properties of wide variety of ordinary, functional and partial equations. Nevertheless, the application of this method to problem of stability in differential equations with delay has encountered serious difficulties if the delay is unbounded or if the equation has unbounded term. It has been noticed that some of theses difficulties vanish by using the fixed point technic. Other advantages of fixed point theory over Lyapunov's method is that the conditions of the former are average while those of the latter are pointwise (see 2, , 1, 9, 10, 11, 12, 15, 17, and references therein).

In paper, we consider the following neutral nonlinear dynamic equations with variable delays given by

$$
x^{\triangle}(t)=-a(t) x^{\sigma}(t)+b(t) g(x(t))+c(t) f\left(x^{\widetilde{\Delta}}\left(t-\tau_{1}(t)\right)\right)+q\left(t, x(t), x\left(t-\tau_{2}(t)\right)\right),
$$

with the initial condition

$$
x(t)=\varphi(t), t \in\left[d_{t_{0}}, t_{0}\right] \cap \mathbb{T},
$$

where

$$
d_{t_{0}}=\inf _{t \in\left[t_{0}, \infty\right) \cap \mathbb{T}}\left\{t-\tau_{1}(t), t-\tau_{2}(t)\right\}
$$

for each $t_{0} \in[0, \infty) \cap \mathbb{T}$ and $\mathbb{T}$ is an unbounded above and below time scale and such that $t_{0} \in \mathbb{T}$.

Our results are obtained with no need of further assumptions on the delta-differentiable of the neutral coefficient $c$ and the twice delta-differentiable of $\tau_{i}$ with $\tau_{i}^{\triangle}(t) \neq 1$ for $t \in[0, \infty) \cap \mathbb{T}$, so that for a given initial function $\varphi \in \Phi_{t_{0}}$ a mapping $P$ for (0.1) is constructed in such a way to map a, carefully chosen, closed convex nonempty subset $\mathrm{D}$ of a Banach space $\mathrm{X}$ into itself on which $\mathrm{P}$ is a contraction mapping possessing a fixed point. This procedure will enable us to establish and prove by means of the contraction mapping theorem ([16], p. 2) the global asymptotic stability in $C_{r d}^{1}$ for the zero solution of (0.1) with a less restrictive conditions. In the special case $\mathbb{T}=\mathbb{R}$, Yazgan, Tunc and Atan in [17] show that the zero solution of (0.1) is globally asymptotically stable in $\mathrm{C}_{\mathrm{rd}}^{1}$ by using the contraction mapping theorem. Then, the results obtained here extend the work of Yazgan, Tunc and Atan [17. 


\section{Preliminaries}

In this section, we consider some advanced topics in the theory of dynamic equations on a time scales. Again, we remind that for a review of this topic we direct the reader to the monographs of Bohner and Peterson [7] and [8].

A time scale $\mathbb{T}$ is a closed nonempty subset of $\mathbb{R}$. For $t \in \mathbb{T}$ the forward jump operator $\sigma$, and the backward jump operator $\rho$, respectively, are defined as $\sigma(t)=\inf \{s \in \mathbb{T}: s>t\}$ and $\rho(t)=\sup \{t \in \mathbb{T}: s<t\}$. These operators allow elements in the time scale to be classified as follows. We say $t$ is right scattered if $\sigma(t)>t$ and right dense if $\sigma(t)=t$. We say $t$ is left scattered if $\rho(t)<t$ and left dense if $\rho(t)=t$. The graininess function $\mu: \mathbb{T} \rightarrow[0, \infty)$, is defined by $\mu(t)=\sigma(t)-t$ and gives the distance between an element and its successor. We set $\inf \emptyset=\sup \mathbb{T}$ and $\sup \emptyset=\inf \mathbb{T}$. If $\mathbb{T}$ has a left scattered maximum $M$, we define $\mathbb{T}^{k}=\mathbb{T} \backslash\{M\}$. Otherwise, we define $\mathbb{T}^{k}=\mathbb{T}$. If $\mathbb{T}$ has a right scattered minimum $m$, we define $\mathbb{T}_{k}=\mathbb{T} \backslash\{m\}$. Otherwise, we define $\mathbb{T}_{k}=\mathbb{T}$.

Let $t \in \mathbb{T}^{k}$ and let $f: \mathbb{T} \rightarrow \mathbb{R}$. The delta derivative of $f(t)$, denoted $f^{\triangle}(t)$, is defined to be the number (when it exists), with the property that, for each $\epsilon>0$, there is a neighborhood $\mathrm{U}$ of $\mathrm{t}$ such that

$$
\left|f(\sigma(t))-f(s)-f^{\triangle}(t)[\sigma(t)-s]\right| \leq \epsilon|\sigma(t)-s|,
$$

for all $s \in U$. If $\mathbb{T}=\mathbb{R}$ then $f^{\triangle}(t)=f^{\prime}(t)$ is the usual derivative. If $\mathbb{T}=\mathbb{Z}$ then $f^{\triangle}(t)=\triangle f(t)=$ $f(t+1)-f(t)$ is the forward difference of $f$ at $t$.

A function $f$ is right dense continuous (rd-continuous), $f \in C_{r d}=C_{r d}(\mathbb{T}, \mathbb{R})$, if it is continuous at every right dense point $t \in \mathbb{T}$ and its left-hand limits exist at each left dense point $t \in \mathbb{T}$. The function $f: \mathbb{T} \rightarrow \mathbb{R}$ is differentiable on $\mathbb{T}^{k}$ provided $f^{\triangle}(t)$ exists for all $t \in \mathbb{T}^{k} . f \in C_{r d}^{1}=C_{r d}^{1}(\mathbb{T}, \mathbb{R})$ if $\mathrm{f}^{\triangle} \in \mathrm{C}_{\mathrm{rd}}(\mathbb{T}, \mathbb{R})$.

We are now ready to state some properties of the delta-derivative of $f$. Note $f^{\sigma}(t)=f(\sigma(t))$.

Theorem 1.1 ([7, Theorem 1.20]). Assume $\mathrm{f}, \mathrm{g}: \mathbb{T} \rightarrow \mathbb{R}$ are differentiable at $\mathrm{t} \in \mathbb{T}^{\mathrm{k}}$ and let $\alpha$ be a scalar.

(i) $(f+g)^{\triangle}(t)=g^{\triangle}(t)+f^{\triangle}(t)$.

(ii) $(\alpha f)^{\triangle}(t)=\alpha f^{\triangle}(t)$.

(iii) The product rules

$$
\begin{aligned}
& (f g)^{\triangle}(t)=f^{\triangle}(t) g(t)+f^{\sigma}(t) g^{\triangle}(t), \\
& (f g)^{\triangle}(t)=f(t) g^{\triangle}(t)+f^{\triangle}(t) g^{\sigma}(t) .
\end{aligned}
$$

(iv) If $\mathrm{g}(\mathrm{t}) \mathrm{g}^{\sigma}(\mathrm{t}) \neq 0$ then

$$
\left(\frac{f}{g}\right)^{\triangle}(t)=\frac{f^{\triangle}(t) g(t)-f(t) g^{\triangle}(t)}{g(t) g^{\sigma}(t)}
$$


The next theorem is the chain rule on time scales ([7, Theorem 1.93]).

Theorem 1.2 (Chain Rule). Assume $v: \mathbb{T} \rightarrow \mathbb{R}$ is strictly increasing and $\widetilde{\mathbb{T}}:=v(\mathbb{T})$ is a time scale. Let $\omega: \widetilde{\mathbb{T}} \rightarrow \mathbb{R}$. If $v^{\triangle}(\mathrm{t})$ and $\omega^{\widetilde{\Delta}}(v(\mathrm{t}))$ exist for $\mathrm{t} \in \mathbb{T}^{k}$, then $(\omega \circ v)^{\triangle}=\left(\omega^{\widetilde{\triangle}} \circ v\right) v^{\triangle}$.

In the sequel we will need to differentiate and integrate functions of the form $f(t-\tau(t))=$ $f(v(t))$ where, $v(t):=t-\tau(t)$. Our next theorem is the substitution rule ([7, Theorem 1.98]).

Theorem 1.3 (Substitution). Assume $v: \mathbb{T} \rightarrow \mathbb{R}$ is strictly increasing and $\widetilde{\mathbb{T}}:=v(\mathrm{~T})$ is a time scale. If $\mathrm{f}: \mathbb{T} \rightarrow \mathbb{R}$ is rd-continuous function and $\boldsymbol{v}$ is differentiable with rd-continuous derivative, then for $\mathrm{a}, \mathrm{b} \in \mathrm{T}$,

$$
\int_{a}^{b} f(t) v^{\triangle}(t) \triangle t=\int_{v(a)}^{v(b)}\left(f \circ v^{-1}\right)(s) \widetilde{\triangle} .
$$

A function $p: \mathbb{T} \rightarrow \mathbb{R}$ is said to be regressive provided $1+\mu(t) p(t) \neq 0$ for all $t \in \mathbb{T}^{k}$. The set of all regressive rd-continuous function $f: \mathbb{T} \rightarrow \mathbb{R}$ is denoted by $\mathcal{R}$. The set of all positively regressive functions $\mathcal{R}^{+}$, is given by $\mathcal{R}^{+}=\{\mathrm{f} \in \mathcal{R}: 1+\mu(\mathrm{t}) \mathrm{f}(\mathrm{t})>0$ for all $\mathrm{t} \in \mathbb{T}\}$.

Let $p \in \mathcal{R}$ and $\mu(t) \neq 0$ for all $t \in \mathbb{T}$. The exponential function on $\mathbb{T}$ is defined by

$$
e_{p}(t, s)=\exp \left(\int_{s}^{t} \frac{1}{\mu(z)} \log (1+\mu(z) p(z)) \Delta z\right) .
$$

It is well known that if $p \in \mathcal{R}^{+}$, then $e_{p}(t, s)>0$ for all $t \in \mathbb{T}$. Also, the exponential function $y(t)=e_{p}(t, s)$ is the solution to the initial value problem $y^{\Delta}=p(t) y, y(s)=1$. Other properties of the exponential function are given by the following lemma.

Lemma 1.4 ([7, Theorem 2.36]). Let $\mathrm{p}, \mathrm{q} \in \mathcal{R}$. Then

(i) $e_{0}(t, s)=1$ and $e_{p}(t, t)=1$,

(ii) $e_{p}(\sigma(t), s)=(1+\mu(t) p(t)) e_{p}(t, s)$

(iii) $\frac{1}{e_{p}(t, s)}=e_{\ominus p}(t, s)$, where $\ominus p(t)=-\frac{p(t)}{1+\mu(t) p(t)}$,

(iv) $e_{p}(t, s)=\frac{1}{e_{p}(s, t)}=e_{\ominus p}(s, t)$,

(v) $e_{p}(t, s) e_{p}(s, r)=e_{p}(t, r)$,

(vi) $e_{\mathrm{p}}^{\triangle}(., s)=p e_{\mathrm{p}}(., s)$ and $\left(\frac{1}{e_{\mathfrak{p}}(., s)}\right)^{\triangle}=-\frac{p(t)}{e_{\mathfrak{p}}^{\sigma}(., s)}$.

Lemma 1.5 ([1]). If $\mathrm{p} \in \mathcal{R}^{+}$, then

$$
0<e_{p}(t, s) \leq \exp \left(\int_{s}^{t} p(u) \Delta u\right), \forall t \in \mathbb{T} .
$$

\section{Global asymptotic stability}

In this section, we shall study the global asymptotic stability in $\mathrm{C}_{\mathrm{rd}}^{1}$ of the zero solution to (0.1). We introduce the following hypotheses. 
$\left(\mathrm{H}_{1}\right) \mathrm{a}, \mathrm{b}, \mathrm{c} \in \mathrm{C}_{\mathrm{rd}}([0, \infty) \cap \mathbb{T}, \mathbb{R}), g, f \in \mathrm{C}(\mathbb{R}, \mathbb{R}), \quad \mathrm{q} \in \mathrm{C}_{\mathrm{rd}}([0, \infty) \cap \mathbb{T} \times \mathbb{R} \times \mathbb{R}, \mathbb{R}), \tau_{\mathrm{i}} \in$ $\mathrm{C}_{\mathrm{rd}}([0, \infty) \cap \mathbb{T},(0, \infty) \cap \mathbb{T})$ and $\left(\mathrm{id}-\tau_{\mathrm{i}}\right)([0, \infty) \cap \mathbb{T})$ is closed with $\mathrm{t}-\tau_{\mathrm{i}}(\mathrm{t}) \rightarrow \infty$ as $\mathrm{t} \rightarrow \infty$, $i=1,2$.

$\left(\mathrm{H}_{2}\right)$ For $\mathrm{t} \in[0, \infty) \cap \mathbb{T}, \mathrm{g}(0)=\mathrm{f}(0)=\mathrm{q}(\mathrm{t}, 0,0)=0$, and there exist $\mathrm{L}_{\mathrm{g}}, \mathrm{L}_{\mathrm{f}}>0, \mathrm{~L}_{1}, \mathrm{~L}_{2} \in$ $\mathrm{C}_{\mathrm{rd}}([0, \infty) \cap \mathbb{T},(0, \infty))$ such that

$$
\begin{gathered}
\left|g\left(x_{1}\right)-g\left(x_{2}\right)\right| \leq L_{g}\left|x_{1}-x_{2}\right|, \\
\left|f\left(x_{1}\right)-f\left(x_{2}\right)\right| \leq L_{f}\left|x_{1}-x_{2}\right|, \\
\left|q\left(t, x_{1}, y_{1}\right)-q\left(t, x_{2}, y_{2}\right)\right| \leq L_{1}(t)\left|x_{1}-x_{2}\right|+L_{2}(t)\left|y_{1}-y_{2}\right|,
\end{gathered}
$$

for any $x_{i}, y_{i} \in \mathbb{R}, i=1,2$.

$\left(\mathrm{H}_{3}\right) \mathrm{a} \in \mathcal{R}^{+}$is bounded on $[0, \infty) \cap \mathbb{T}$ and $\lim _{\mathrm{t} \rightarrow \infty} \inf \int_{0}^{\mathrm{t}} \frac{1}{\mu(\mathrm{s})} \log (1+\mu(\mathrm{s}) \mathrm{a}(\mathrm{s})) \Delta \mathrm{s}>-\infty$.

$\left(\mathrm{H}_{4}\right)$ There exists $\alpha \in(0,1)$ such that for $\mathrm{t} \in[0, \infty) \cap \mathbb{T}$,

$$
\int_{0}^{t} e_{\ominus a}(t, u)\left[L_{g}|b(u)|+L_{f}|c(u)|+L_{1}(u)+L_{2}(u)\right] \Delta u \leq \alpha,
$$

and

$$
\begin{aligned}
& |a(t)| \int_{0}^{\sigma(t)} e_{\ominus a}(\sigma(t), u)\left[L_{g}|b(u)|+L_{f}|c(u)|+L_{1}(u)+L_{2}(u)\right] \Delta u \\
& +L_{g}|b(t)|+L_{f}|c(t)|+L_{1}(t)+L_{2}(t) \leq \alpha .
\end{aligned}
$$

For each $t_{0} \in[0, \infty) \cap \mathbb{T}$ denote $C_{r d}^{1}\left(t_{0}\right)=C_{r d}^{1}\left(\left[d_{t_{0}}, t_{0}\right] \cap \mathbb{T}, \mathbb{R}\right)$ with the norm defined by

$$
|x|_{t_{0}}=\max _{t \in\left[d_{t_{0}}, t_{0}\right] \cap \mathbb{T}}\left\{|x(t)|,\left|x^{\triangle}(t)\right|\right\}
$$

for $x \in C_{r d}^{1}\left(t_{0}\right)$. In addition, denote

$$
\begin{aligned}
\Phi_{t_{0}} & =\left\{\varphi \in C_{r d}^{1}\left(t_{0}\right): \varphi^{\triangle}\left(t_{0}\right)=-a\left(t_{0}\right) \varphi^{\sigma}\left(t_{0}\right)+b\left(t_{0}\right) g\left(\varphi\left(t_{0}\right)\right)\right. \\
& \left.+c\left(t_{0}\right) f\left(\varphi^{\widetilde{\Delta}}\left(t_{0}-\tau_{1}\left(t_{0}\right)\right)\right)+q\left(t_{0}, \varphi\left(t_{0}\right), \varphi\left(t_{0}-\tau_{2}\left(t_{0}\right)\right)\right)\right\} .
\end{aligned}
$$

For each $t_{0} \in[0, \infty) \cap \mathbb{T}$, we always assume that the initial function for (0.1) is of the type $\varphi \in \Phi_{t_{0}}$. For convenience of stating our main result, we shall give the following definitions.

Definition 2.1. For each $\left(\mathrm{t}_{0}, \varphi\right) \in[0, \infty) \cap \mathbb{T} \times \Phi_{\mathrm{t}_{0}}, x$ is said to be a solution of (0.1) through $\left(\mathrm{t}_{0}, \varphi\right)$ if $\mathrm{x} \in \mathrm{C}_{\mathrm{rd}}^{1}\left(\left[\mathrm{~d}_{\mathrm{t}_{0}}, \infty\right) \cap \mathbb{T}\right)$ satisfies (0.1) on $\left[\mathrm{t}_{0}, \infty\right) \cap \mathbb{T}$ and $\mathrm{x}(\mathrm{t})=\varphi(\mathrm{t})$ for $\mathrm{t} \in\left[\mathrm{d}_{\mathrm{t}_{0}}, \mathrm{t}_{0}\right] \cap \mathbb{T}$. We denote such a solution by $\mathrm{x}(\mathrm{t})=\mathrm{x}\left(\mathrm{t}, \mathrm{t}_{\mathrm{o}}, \varphi\right)$.

Definition 2.2. (i) The zero solution of (0.1) is said to be stable in $\mathrm{C}_{\mathrm{rd}}^{1}$ if, for any $\mathrm{t}_{\mathrm{o}} \in[0, \infty) \cap \mathbb{T}$, $\varepsilon>0$ there is a $\delta=\delta\left(\varepsilon, t_{0}\right)$ such that $\varphi \in \Phi_{t_{0}}$ and $|\varphi|_{t_{0}}<\delta$ implies

$$
\max _{s \in\left[\mathrm{d}_{\mathrm{t}_{0}}, \mathrm{t}\right] \cap \mathbb{T}}\left\{|x(\mathrm{~s})|,\left|\mathrm{x}^{\triangle}(\mathrm{s})\right|\right\}<\varepsilon,
$$


for $\mathrm{t} \in\left[\mathrm{t}_{0}, \infty\right) \cap \mathbb{T}$.

(ii) The zero solution of (0.1) is said to be globally asymptotically stable in $\mathrm{C}_{\mathrm{rd}}^{1}$ if it is stable in $\mathrm{C}_{\mathrm{rd}}^{1}$, and for any $\mathrm{t}_{\mathrm{o}} \in[0, \infty) \cap \mathbb{T}, \varphi \in \Phi_{\mathrm{t}_{\mathrm{o}}}$ implies

$$
\lim _{t \rightarrow \infty} x\left(t, t_{0}, \varphi\right)=\lim _{t \rightarrow \infty} x^{\triangle}\left(t, t_{0}, \varphi\right)=0 .
$$

In view of the definition of solution of (0.1), it is clear that the conditions imposed on the initial functions are very natural. From the above assumptions, it is easy to see that for each $\left(t_{0}, \varphi\right) \in[0, \infty) \cap \mathbb{T} \times \Phi_{t_{0}}$, there exists a unique solution $x(t)=x\left(t, t_{0}, \varphi\right)$ of (0.1) defined on $\left[\mathrm{d}_{\mathrm{t}_{0}}, \infty\right) \cap \mathbb{T}$. By $\left(\mathrm{H}_{2}\right)$, (0.1) has the zero solution.

Theorem 2.3. Assume that $\left(\mathrm{H}_{1}\right)-\left(\mathrm{H}_{4}\right)$ hold. Then the zero solution of (0.1) is globally asymptotically stable in $\mathrm{C}_{\mathrm{rd}}^{1}$ if and only if

$$
\int_{0}^{t} \frac{1}{\mu(s)} \log (1+\mu(s) a(s)) \Delta s \rightarrow \infty \text { as } \mathrm{t} \rightarrow \infty .
$$

Proof. (i) Suppose that (2.1) holds. For any $t_{0} \in[0, \infty) \cap \mathbb{T}$, let

$$
X=\left\{x \in C_{r d}^{1}\left(\left[d_{t_{0}}, \infty\right) \cap \mathbb{T}\right): \lim _{t \rightarrow \infty} x(t)=\lim _{t \rightarrow \infty} x^{\triangle}(t)=0\right\}
$$

with the norm defined by

$$
\|x\|_{t_{0}}=\max _{t \in\left[d_{t_{0}}, \infty\right) \cap \mathbb{T}}\left\{|x(t)|,\left|x^{\triangle}(t)\right|\right\},
$$

for $x \in X$. Since $X$ is a closed vectorial subspace of $C_{r d}^{1}\left(\left[d_{t_{0}}, \infty\right) \cap \mathbb{T}\right)$ and $C_{r d}^{1}\left(\left[d_{t_{0}}, \infty\right) \cap \mathbb{T},\|\cdot\|_{t_{0}}\right)$ is a Banach space, then $\left(X,\|\cdot\|_{t_{0}}\right)$ is also a Banach space. For any $\varphi \in \Phi_{t_{0}}$, let

$$
\mathrm{D}=\left\{x \in X: x(t)=\varphi(t) \text { for } t \in\left[d_{t_{0}}, t_{0}\right] \cap \mathbb{T}\right\} .
$$

It is easy to see that $\mathrm{D}$ is a nonempty, closed subset of $\mathrm{X}$.

Multiply both sides of (0.1) by $e_{a}\left(t, t_{0}\right)$ and then integrate from $t_{0}$ to $t$ to obtain

$$
\begin{aligned}
& \int_{t_{0}}^{t}\left[x(u) e_{a}\left(u, t_{0}\right)\right]^{\Delta} \Delta u \\
& =\int_{t_{0}}^{t} e_{a}\left(u, t_{0}\right)\left[b(u) g(x(u))+c(u) f\left(x^{\widetilde{\Delta}}\left(u-\tau_{1}(u)\right)\right)\right. \\
& \left.+q\left(u, x(u), x\left(u-\tau_{2}(u)\right)\right)\right] \Delta u .
\end{aligned}
$$

As a consequence, we arrive at

$$
\begin{aligned}
& x(t) e_{a}\left(t, t_{0}\right)-x\left(t_{0}\right) \\
& =\int_{t_{0}}^{t} e_{a}\left(u, t_{0}\right)\left[b(u) g(x(u))+c(u) f\left(x^{\widetilde{\Delta}}\left(u-\tau_{1}(u)\right)\right)\right. \\
& \left.+q\left(u, x(u), x\left(u-\tau_{2}(u)\right)\right)\right] \Delta u .
\end{aligned}
$$


By dividing both sides of the above equation by $e_{a}\left(t, t_{0}\right)$, we obtain

$$
\begin{aligned}
x(t) & =\varphi\left(t_{0}\right) e_{\ominus a}\left(t, t_{0}\right)+\int_{t_{0}}^{t} e_{\ominus a}(t, u)\left[b(u) g(x(u))+c(u) f\left(x^{\widetilde{\Delta}}\left(u-\tau_{1}(u)\right)\right)\right. \\
& \left.+q\left(u, x(u), x\left(u-\tau_{2}(u)\right)\right)\right] \Delta u .
\end{aligned}
$$

Use (2.2) to define the operator $P: D \rightarrow C_{r_{d}}\left(\left[d_{t_{0}}, \infty\right) \cap \mathbb{T}\right)$ by $(P x)(t)=\varphi(t)$ for $t \in\left[d_{t_{0}}, t_{0}\right] \cap \mathbb{T}$ and

$$
\begin{aligned}
(P x)(t) & =\varphi\left(t_{0}\right) e_{\ominus a}\left(t, t_{0}\right)+\int_{t_{0}}^{t} e_{\ominus a}(t, u)\left[b(u) g(x(u))+c(u) f\left(x^{\widetilde{\Delta}}\left(u-\tau_{1}(u)\right)\right)\right. \\
& \left.+q\left(u, x(u), x\left(u-\tau_{2}(u)\right)\right)\right] \Delta u
\end{aligned}
$$

for $t \in\left[t_{0}, \infty\right) \cap \mathbb{T}$.

Firstly, we prove $\mathrm{P} x \in \mathrm{D}$ for any $x \in \mathrm{D}$. From (2.3), for $\mathrm{t}>\mathrm{t}_{0}$,

$$
\begin{aligned}
(P x)^{\triangle}(t) & =-\varphi\left(t_{0}\right) a(t) e_{\ominus a}\left(\sigma(t), t_{0}\right) \\
& +e_{\ominus a}(\sigma(t), t)\left[b(t) g(x(t))+c(t) f\left(x^{\widetilde{\Delta}}\left(t-\tau_{1}(t)\right)\right)+q\left(t, x(t), x\left(t-\tau_{2}(t)\right)\right)\right] \\
& -a(t) \int_{t_{0}}^{t} e_{\ominus a}(\sigma(t), u)\left[b(u) g(x(u))+c(u) f\left(x^{\widetilde{\Delta}}\left(u-\tau_{1}(u)\right)\right)\right. \\
& \left.+q\left(u, x(u), x\left(u-\tau_{2}(u)\right)\right)\right] \Delta u \\
& =-a(t) \varphi\left(t_{0}\right) e_{\ominus a}\left(\sigma(t), t_{0}\right) \\
& -a(t) \int_{t_{0}}^{\sigma(t)} e_{\ominus a}(\sigma(t), u)\left[b(u) g(x(u))+c(u) f\left(x^{\widetilde{\Delta}}\left(u-\tau_{1}(u)\right)\right)\right. \\
& \left.+q\left(u, x(u), x\left(u-\tau_{2}(u)\right)\right)\right] \Delta u \\
& +b(t) g(x(t))+c(t) f\left(x^{\widetilde{\Delta}}\left(t-\tau_{1}(t)\right)\right)+q\left(t, x(t), x\left(t-\tau_{2}(t)\right)\right) \\
& =-a(t)(P x)^{\sigma}(t)+b(t) g(x(t))+c(t) f\left(x^{\widetilde{\Delta}}\left(t-\tau_{1}(t)\right)\right)+q\left(t, x(t), x\left(t-\tau_{2}(t)\right)\right) .
\end{aligned}
$$

By the definition of $\Phi_{t_{0}},(2.4)$ yields on a time scale

$$
\begin{aligned}
(P x)_{+}^{\triangle}\left(t_{0}\right) & =-a\left(t_{0}\right) \varphi^{\sigma}\left(t_{0}\right)+b\left(t_{0}\right) g\left(\varphi\left(t_{0}\right)\right)+c\left(t_{0}\right) f\left(\varphi^{\widetilde{\Delta}}\left(t_{0}-\tau_{1}\left(t_{0}\right)\right)\right) \\
& +q\left(t_{0}, \varphi\left(t_{0}\right), \varphi\left(t_{0}-\tau_{2}\left(t_{0}\right)\right)\right) \\
& =\varphi_{-}^{\triangle}\left(t_{0}\right) .
\end{aligned}
$$

Hence, $\mathrm{P} x \in \mathrm{C}_{\mathrm{rd}}^{1}\left(\left[\mathrm{~d}_{\mathrm{t}_{0}}, \infty\right) \cap \mathbb{T}\right)$ for $x \in \mathrm{D}$.

For $x \in D, \lim _{t \rightarrow \infty} x(t)=\lim _{t \rightarrow \infty} x^{\triangle}(t)=0$. Note that $\lim _{t \rightarrow \infty} t-\tau_{i}(t)=\infty, i=1,2$. Therefore, for any $\varepsilon>0$, there exists $T>0$ such that for $t \geq T$,

$$
\max \left\{|x(t)|,\left|x\left(t-\tau_{2}(t)\right)\right|,\left|x^{\widetilde{\Delta}}\left(t-\tau_{1}(t)\right)\right|\right\}<\varepsilon .
$$


It follows from (2.3), (2.5) and $\left(\mathrm{H}_{2}\right)$ and $\left(\mathrm{H}_{4}\right)$ that for $\mathrm{t}>\mathrm{T}$ and $\mathrm{x} \in \mathrm{D}$,

$$
\begin{aligned}
& |(P x)(t)| \\
& \leq\left|\varphi\left(t_{0}\right)\right| e_{\ominus a}\left(t, t_{0}\right)+\int_{t_{0}}^{T} e_{\ominus a}(t, u) \mid b(u) g(x(u))+c(u) f\left(x^{\widetilde{\triangle}}\left(u-\tau_{1}(u)\right)\right) \\
& +q\left(u, x(u), x\left(u-\tau_{2}(u)\right)\right) \mid \Delta u \\
& +\int_{T}^{t} e_{\ominus a}(t, u) \mid b(u)(g(x(u))-g(0))+c(u)\left(f\left(x^{\widetilde{\Delta}}\left(u-\tau_{1}(u)\right)\right)-f(0)\right) \\
& +q\left(u, x(u), x\left(u-\tau_{2}(u)\right)\right)-q(u, 0,0) \mid \Delta u \\
& \leq e_{\ominus a}\left(t, t_{0}\right)\left[\left|\varphi\left(t_{0}\right)\right|+\int_{t_{0}}^{T} e_{a}\left(u, t_{0}\right) \mid b(u) g(x(u))+c(u) f\left(x^{\widetilde{\triangle}}\left(u-\tau_{1}(u)\right)\right)\right. \\
& \left.+q\left(u, x(u), x\left(u-\tau_{2}(u)\right)\right) \mid \Delta u\right] \\
& +\int_{T}^{t} e_{\ominus a}(t, u)\left[L_{g}|b(u)||x(u)|+L_{f}|c(u)|\left|x^{\widetilde{\triangle}}\left(u-\tau_{1}(u)\right)\right|\right. \\
& \left.+\mathrm{L}_{1}(\mathrm{u})|x(\mathrm{u})|+\mathrm{L}_{2}(\mathrm{u})\left|\chi\left(\mathrm{u}-\tau_{2}(\mathrm{u})\right)\right|\right] \Delta \mathrm{u} \\
& \leq e_{\ominus a}\left(t, t_{0}\right)\left[\left|\varphi\left(t_{0}\right)\right|+\int_{t_{0}}^{T} e_{a}\left(u, t_{0}\right) \mid b(u) g(x(u))+c(u) f\left(x^{\widetilde{\triangle}}\left(u-\tau_{1}(u)\right)\right)\right. \\
& \left.+q\left(u, x(u), x\left(u-\tau_{2}(u)\right)\right) \mid \Delta u\right] \\
& +\varepsilon \int_{T}^{t} e_{\ominus a}(t, u)\left[L_{g}|b(u)|+L_{f}|c(u)|+L_{1}(u)+L_{2}(u)\right] \Delta u \\
& \leq e_{\ominus a}\left(t, t_{0}\right)\left[\left|\varphi\left(t_{0}\right)\right|+\int_{t_{0}}^{T} e_{a}\left(u, t_{0}\right) \mid b(u) g(x(u))+c(u) f\left(x^{\widetilde{\triangle}}\left(u-\tau_{1}(u)\right)\right)\right. \\
& \left.+q\left(u, x(u), x\left(u-\tau_{2}(u)\right)\right) \mid \Delta u\right]+\alpha \varepsilon \text {. }
\end{aligned}
$$

From (2.1), there exists $\mathrm{T}_{1}>\mathrm{T}$ such that for $\mathrm{t}>\mathrm{T}_{1}$,

$$
\begin{aligned}
& e_{\ominus a}\left(t, t_{0}\right)\left[\left|\varphi\left(t_{0}\right)\right|+\int_{t_{0}}^{T} e_{a}\left(u, t_{0}\right) \mid b(u) g(x(u))+c(u) f\left(x^{\widetilde{\Delta}}\left(u-\tau_{1}(u)\right)\right)\right. \\
& \left.+q\left(u, x(u), x\left(u-\tau_{2}(u)\right)\right) \mid \Delta u\right]<\varepsilon .
\end{aligned}
$$

Hence, $\lim _{t \rightarrow \infty}(P x)(t)=0$ for $x \in D$. In addition, it follows from (2.4) and $\left(\mathrm{H}_{2}\right)$ that

$$
\begin{aligned}
& \left|(P x)^{\triangle}(t)\right| \\
& \leq\left|a(t)(P x)^{\sigma}(t)\right|+|b(t)(g(x(t))-g(0))|+\left|c(t)\left(f\left(x^{\widetilde{\triangle}}\left(t-\tau_{1}(t)\right)\right)-f(0)\right)\right| \\
& +\left|q\left(t, x(t), x\left(t-\tau_{2}(t)\right)\right)-q(t, 0,0)\right| \\
& \leq\left|a(t)(P x)^{\sigma}(t)\right|+L_{g}|b(t)||x(t)|+L_{f}|c(t)|\left|x^{\widetilde{\triangle}}\left(t-\tau_{1}(t)\right)\right| \\
& +L_{1}(t)|x(t)|+L_{2}(t)\left|x\left(t-\tau_{2}(t)\right)\right| .
\end{aligned}
$$


This, together with $\left(\mathrm{H}_{3}\right)$ and $\left(\mathrm{H}_{4}\right)$, yields $\lim _{t \rightarrow \infty}(\mathrm{Px})^{\triangle}(\mathrm{t})=0$ for $x \in \mathrm{D}$. Therefore, $\mathrm{P} x \in \mathrm{D}$ for $x \in \mathrm{D}$, i.e. $\mathrm{P}: \mathrm{D} \rightarrow \mathrm{D}$.

Secondly, we show that $\mathrm{P}: \mathrm{D} \rightarrow \mathrm{D}$ is a contraction mapping. For any $x, y \in D$, it follows from (2.3), $\left(\mathrm{H}_{2}\right)$ and $\left(\mathrm{H}_{4}\right)$ that for $\mathrm{t} \in\left[\mathrm{t}_{0}, \infty\right) \cap \mathbb{T}$,

$$
\begin{aligned}
& |(P x)(t)-(P y)(t)| \\
& \leq \int_{t_{0}}^{t} e_{\ominus a}(t, u)\left[L_{g}|b(u)||x(u)-y(u)|+L_{f}|c(u)|\left|x^{\widetilde{\Delta}}\left(u-\tau_{1}(u)\right)-y^{\widetilde{\Delta}}\left(u-\tau_{1}(u)\right)\right|\right. \\
& \left.+\left|q\left(u, x(u), x\left(u-\tau_{2}(u)\right)\right)-q\left(u, y(u), y\left(u-\tau_{2}(u)\right)\right)\right|\right] \Delta u \\
& \leq\|x-y\|_{t_{0}} \int_{t_{0}}^{t} e_{\ominus a}(t, u)\left[L_{g}|b(u)|+L_{f}|c(u)|+L_{1}(u)+L_{2}(u)\right] \Delta u \\
& \leq \alpha\|x-y\|_{t_{0}} .
\end{aligned}
$$

In addition, it follows from (2.4), (2.6), $\left(\mathrm{H}_{2}\right)$ and $\left(\mathrm{H}_{4}\right)$ that for $\mathrm{t} \in\left[\mathrm{t}_{0}, \infty\right) \cap \mathbb{T}$,

$$
\begin{aligned}
& \left|(P x)^{\triangle}(t)-(P y)^{\triangle}(t)\right| \\
& \leq|a(t)|\left|(P x)^{\sigma}(t)-(P y)^{\sigma}(t)\right|+L_{g}|b(t)||x(t)-y(t)|+L_{f}|c(t)|\left|x^{\widetilde{\Delta}}\left(t-\tau_{1}(t)\right)-y^{\widetilde{\Delta}}\left(t-\tau_{1}(t)\right)\right| \\
& +\left|q\left(t, x(t), x\left(t-\tau_{2}(t)\right)\right)-q\left(t, y(t), y\left(t-\tau_{2}(t)\right)\right)\right| \\
& \leq\|x-y\|_{t_{0}}\left[|a(t)| \int_{t_{0}}^{\sigma(t)} e_{\ominus a}(\sigma(t), u)\left[L_{g}|b(u)|+L_{f}|c(u)|+L_{1}(u)+L_{2}(u)\right] \Delta u\right. \\
& \left.+L_{g}|b(t)|+L_{f}|c(t)|+L_{1}(t)+L_{2}(t)\right] \\
& \leq \alpha\|x-y\|_{t_{0}} .
\end{aligned}
$$

From (2.6) and (2.7), $\mathrm{P}: \mathrm{D} \rightarrow \mathrm{D}$ is a contraction mapping. By the contraction mapping principle, $P$ has a unique fixed point $x$ in $D$, which is a unique solution of (0.1) through $\left(t_{0}, \varphi\right)$ and satisfies

$$
\lim _{t \rightarrow \infty} x(t)=\lim _{t \rightarrow \infty} x^{\triangle}(t)=0 .
$$

Finally, we show that the zero solution of (0.1) is stable in $\mathrm{C}_{\mathrm{rd}}^{1}$. Let

$$
\mathrm{K}=\sup _{\mathrm{t} \in\left[\mathrm{t}_{0}, \infty\right) \cap \mathbb{T}}\left\{\boldsymbol{e}_{\ominus \mathbf{a}}\left(\mathrm{t}, \mathrm{t}_{0}\right)\right\} \text { and } A=\sup _{\mathrm{t} \in\left[\mathrm{t}_{0}, \infty\right) \cap \mathbb{T}}\{|\mathbf{a}(\mathrm{t})|\} .
$$

From (2.1) and $\left(\mathrm{H}_{3}\right), \mathrm{K}, \mathrm{A} \in(0, \infty)$. For any $\varepsilon>0$, let $\delta>0$ such that

$$
\delta<\varepsilon \min \left\{1, \frac{1-\alpha}{\mathrm{K}}, \frac{1-\alpha}{\mathrm{KA}}\right\} .
$$

If $x(t)=x\left(t, t_{0}, \varphi\right)$ is a solution of (0.1) with $|\varphi|_{t_{0}}<\delta$, then $x(t)=(P x)(t)$ on $\left[t_{0}, \infty\right) \cap \mathbb{T}$. We claim that $\|x\|_{t_{0}}<\varepsilon$. Otherwise, there exists $t_{1}>t_{0}$ such that

$$
\max \left\{\left|x\left(t_{1}\right)\right|,\left|x^{\triangle}\left(t_{1}\right)\right|\right\}=\varepsilon
$$


and

$$
\max \left\{|x(t)|,\left|x^{\triangle}(t)\right|\right\}<\varepsilon,
$$

for $t \in\left[d_{t_{0}}, t_{1}\right) \cap \mathbb{T}$. If $\left|x\left(t_{1}\right)\right|=\varepsilon$, then it follows from (2.3), $\left(H_{2}\right)$ and $\left(H_{4}\right)$ that

$$
\begin{aligned}
\left|x\left(t_{1}\right)\right| & \leq\left|\varphi\left(t_{0}\right)\right| e_{\ominus a}\left(t_{1}, t_{0}\right) \\
& +\int_{t_{0}}^{t_{1}} e_{\ominus a}\left(t_{1}, u\right)\left|b(u) g(x(u))+c(u) f\left(x^{\widetilde{\triangle}}\left(u-\tau_{1}(u)\right)\right)+q\left(u, x(u), x\left(u-\tau_{2}(u)\right)\right)\right| \Delta u \\
& \leq K \delta+\varepsilon \int_{t_{0}}^{t_{1}} e_{\ominus a}\left(t_{1}, u\right)\left[L_{g}|b(u)|+L_{f}|c(u)|+L_{1}(u)+L_{2}(u)\right] \Delta u \\
& \leq K \delta+\alpha \varepsilon \\
& <\varepsilon .
\end{aligned}
$$

This is a contradiction. If $\left|x^{\triangle}\left(\mathrm{t}_{1}\right)\right|=\varepsilon$, then it follows from (2.4) and $\left(\mathrm{H}_{2}\right)$ and $\left(\mathrm{H}_{4}\right)$ that

$$
\begin{aligned}
& \left|x^{\triangle}\left(t_{1}\right)\right| \\
& \leq\left|\varphi\left(t_{0}\right) a\left(t_{1}\right)\right| e_{\ominus a}\left(\sigma\left(t_{1}\right), t_{0}\right)+\left|b\left(t_{1}\right)\right|\left|g\left(x\left(t_{1}\right)\right)\right| \\
& +\left|c\left(t_{1}\right)\right| f\left(x^{\widetilde{\Delta}}\left(t_{1}-\tau_{1}\left(t_{1}\right)\right)\right)|+| q\left(t_{1}, x\left(t_{1}\right), x\left(t_{1}-\tau_{2}\left(t_{1}\right)\right)\right) \mid \\
& +\left|a\left(t_{1}\right)\right| \int_{t_{0}}^{\sigma\left(t_{1}\right)} e_{\ominus a}\left(\sigma\left(t_{1}\right), u\right)\left|b(u) g(x(u))+c(u) f\left(x \widetilde{\triangle}\left(u-\tau_{1}(u)\right)\right)+q\left(u, x(u), x\left(u-\tau_{2}(u)\right)\right)\right| \Delta u \\
& \leq K A \delta+\varepsilon\left\{\left|a\left(t_{1}\right)\right| \int_{t_{0}}^{\sigma\left(t_{1}\right)} e_{\ominus a}\left(\sigma\left(t_{1}\right), u\right)\left[L_{g}|b(u)|+L_{f}|c(u)|+L_{1}(u)+L_{2}(u)\right] \Delta u\right. \\
& \left.+L_{g}\left|b\left(t_{1}\right)\right|+L_{f}\left|c\left(t_{1}\right)\right|+L_{1}\left(t_{1}\right)+L_{2}\left(t_{1}\right)\right\} \\
& \leq K A \delta+\alpha \varepsilon \\
& <\varepsilon .
\end{aligned}
$$

This is also a contradiction. Hence, the zero solution of (0.1) is stable in $C_{r d}^{1}$. This, together with (2.8), implies that the zero solution of (0.1) is globally asymptotically stable in $\mathrm{C}_{\mathrm{rd}}^{1}$.

(ii) Assume that the zero solution of (0.1) is globally asymptotically stable in $\mathrm{C}_{\mathrm{rd}}^{1}$. Now we prove that (2.1) holds. Otherwise, set

$$
l=\lim _{t \mapsto \infty} \inf \int_{0}^{t} \frac{1}{\mu(s)} \log (1+\mu(s) a(s)) \Delta s, K_{0}=\sup _{t \in[0, \infty) \cap \mathbb{T}}\left\{e_{\ominus a}(t, 0)\right\}, A_{0}=\sup _{t \in[0, \infty) \cap \mathbb{T}}\{|a(t)|\}
$$

thus it follows from $\left(\mathrm{H}_{3}\right)$ that $l \in(-\infty, \infty), \mathrm{K}_{0} \in(0, \infty), A_{0} \in[0, \infty)$. Hence, there exists an increasing sequence $\left\{t_{n}\right\} \subset[0, \infty) \cap \mathbb{T}$ such that $\lim _{t \rightarrow \infty} t_{n}=\infty$ and

$$
\lim _{n \mapsto \infty} \int_{0}^{t_{n}} \frac{1}{\mu(s)} \log (1+\mu(s) a(s)) \Delta s=l .
$$

\section{Denote}

$$
I_{n}=\int_{0}^{t_{n}} e_{a}(u, 0)\left[L_{g}|b(u)|+L_{f}|c(u)|+L_{1}(u)+L_{2}(u)\right] \Delta u, \quad n=1,2, \ldots .
$$


From $\left(\mathrm{H}_{4}\right)$, it follows that

$$
I_{n}=e_{a}\left(t_{n}, 0\right) \int_{0}^{t_{n}} e_{\ominus a}\left(t_{n}, u\right)\left[L_{g}|b(u)|+L_{f}|c(u)|+L_{1}(u)+L_{2}(u)\right] \Delta u \leq \alpha e_{a}\left(t_{n}, 0\right) .
$$

This, together with (2.9), implies that the sequence $\left\{I_{n}\right\}$ is bounded. Further, there exists a convergent subsequence. For brevity of notation, we may assume that $\left\{\mathrm{I}_{n}\right\}$ is convergent. Therefore, there exists a positive integer $m$ such that for any integer $n>m$,

$$
\int_{t_{m}}^{t_{n}} e_{a}(u, 0)\left[L_{g}|b(u)|+L_{f}|c(u)|+L_{1}(u)+L_{2}(u)\right] \Delta u<\frac{1-\alpha}{8 B\left(e^{-l}+1\right)},
$$

and

$$
e_{\ominus a}\left(t_{n}, t_{m}\right)>\frac{1}{2}, \quad e_{\ominus a}\left(t_{n}, 0\right)<e^{-l}+1, \quad e_{a}\left(t_{m}, 0\right)<e^{l}+1,
$$

where $B=\max \left\{K_{0}\left(e^{l}+1\right), K_{0} A_{0}\left(e^{l}+1\right), 1\right\}$.

For any $\delta>0$, consider the solution $x(t)=x\left(t, t_{m}, \varphi\right)$ of (0.1) with $|\varphi|_{t_{m}}<\delta$ and $\left|\varphi\left(t_{m}\right)\right|>$ $\delta / 2$. It follows from (2.3), (2.4), (2.11), $\left(\mathrm{H}_{2}\right)$ and $\left(\mathrm{H}_{4}\right)$ that for $\mathrm{t} \in\left[\mathrm{t}_{\mathrm{m}}, \infty\right) \cap \mathbb{T}$,

$$
\begin{aligned}
& |x(t)| \\
& \leq\left|\varphi\left(t_{m}\right)\right| e_{\ominus a}\left(t, t_{m}\right) \\
& +\int_{t_{m}}^{t} e_{\ominus a}(t, u)\left|b(u) g(x(u))+c(u) f\left(x^{\widetilde{\Delta}}\left(u-\tau_{1}(u)\right)\right)+q\left(u, x(u), x\left(u-\tau_{2}(u)\right)\right)\right| \Delta u \\
& \leq\left|\varphi\left(t_{m}\right)\right| e_{\ominus a}(t, 0) e_{a}\left(t_{m}, 0\right)+\|x\|_{t_{m}} \int_{t_{m}}^{t} e_{\ominus a}(t, u)\left[L_{g}|b(u)|+L_{f}|c(u)|+L_{1}(u)+L_{2}(u)\right] \Delta u \\
& \leq K_{0}\left(e^{l}+1\right) \delta+\|x\|_{t_{m}} \int_{0}^{t} e_{\ominus a}(t, u)\left[L_{g}|b(u)|+L_{f}|c(u)|+L_{1}(u)+L_{2}(u)\right] \Delta u \\
& \leq B \delta+\alpha\|x\|_{t_{m}},
\end{aligned}
$$

and

$$
\begin{aligned}
& \left|x^{\triangle}(t)\right| \\
& \leq\left|\varphi\left(t_{m}\right)\right||a(t)| e_{\ominus a}\left(\sigma(t), t_{m}\right)+|b(t)||g(x(t))| \\
& +|c(t)|\left|f\left(x^{\widetilde{\Delta}}\left(t-\tau_{1}(t)\right)\right)\right|+\left|q\left(t, x(t), x\left(t-\tau_{2}(t)\right)\right)\right| \\
& +|a(t)| \int_{t_{m}}^{\sigma(t)} e_{\ominus a}(\sigma(t), u)\left|b(u) g(x(u))+c(u) f\left(x^{\widetilde{\Delta}}\left(u-\tau_{1}(u)\right)\right)+q\left(u, x(u), x\left(u-\tau_{2}(u)\right)\right)\right| \Delta u \\
& \leq K_{0} A_{0}\left(e^{l}+1\right) \delta \\
& +\|x\|_{t_{m}}\left\{|a(t)| \int_{t_{m}}^{\sigma(t)} e_{\ominus a}(\sigma(t), u)\left[L_{g}|b(u)|+L_{f}|c(u)|+L_{1}(u)+L_{2}(u)\right] \Delta u\right. \\
& \left.+L_{g}|b(t)|+L_{f}|c(t)|+L_{1}(t)+L_{2}(t)\right\} \\
& \leq B \delta+\alpha\|x\|_{t_{m}} .
\end{aligned}
$$


Hence, $\|x\|_{t_{m}} \leq B \delta+\alpha\|x\|_{t_{m}}$, i.e.

$$
\|x\|_{t_{m}} \leq \frac{B}{1-\alpha} \delta .
$$

It follows from (2.3), (2.10)-(2.12) and $\left(\mathrm{H}_{2}\right)$ that for any $\mathrm{n}>\mathrm{m}$,

$$
\begin{aligned}
& \left|x\left(t_{n}\right)\right| \\
& \geq\left|\varphi\left(t_{m}\right)\right| e_{\ominus a}\left(t_{n}, t_{m}\right) \\
& -e_{\ominus a}\left(t_{n}, 0\right) \int_{t_{m}}^{t_{n}} e_{a}(u, 0)\left|b(u) g(x(u))+c(u) f\left(x^{\widetilde{\Delta}}\left(u-\tau_{1}(u)\right)\right)+q\left(u, x(u), x\left(u-\tau_{2}(u)\right)\right)\right| \Delta u \\
& \geq\left|\varphi\left(t_{m}\right)\right| e_{\ominus a}\left(t_{n}, t_{m}\right)-\|x\|_{t_{m}} e_{\ominus a}\left(t_{n}, 0\right) \int_{t_{m}}^{t_{n}} e_{a}(u, 0)\left[L_{g}|b(u)|+L_{f}|c(u)|+L_{1}(u)+L_{2}(u)\right] \Delta u \\
& >\frac{1}{4} \delta-\frac{B}{1-\alpha} \delta\left(e^{-l}+1\right) \frac{1-\alpha}{8 B\left(e^{-l}+1\right)}=\frac{1}{8} \delta .
\end{aligned}
$$

This contradicts the fact that $\lim _{n \rightarrow \infty} t_{n}=\infty$ and the zero solution of (0.1) is globally asymptotically stable in $\mathrm{C}_{\mathrm{rd}}^{1}$. The proof is complete.

Example 2.4. Let $\mathbb{T}=\mathbb{R}$. Consider the following neutral differential equation

$$
x^{\prime}(t)=-a(t) x(t)+b(t) g(x(t))+c(t) f\left(x^{\prime}\left(t-\tau_{1}(t)\right)\right)+q\left(t, x(t), x\left(t-\tau_{2}(t)\right)\right),
$$

where

$$
\begin{aligned}
\tau_{1}(t) & =t / 2+1, \tau_{2}(t)=t / 3+2, a(t)=\frac{1}{t+1}, b(t)=\frac{1}{15(t+1)}, \\
c(t) & =\frac{1}{12(t+1)}, g(x)=1-\cos (x), f(x)=\sin (x), \\
q(t, x, y) & =\frac{1}{16(t+1)} \sin (x+y) .
\end{aligned}
$$

Obviously a, b, c $\in \mathrm{C}([0, \infty), \mathbb{R}), \mathrm{g}, \mathrm{f} \in \mathrm{C}(\mathbb{R}, \mathbb{R}), \mathrm{q} \in \mathrm{C}([0, \infty) \times \mathbb{R} \times \mathbb{R}, \mathbb{R}), \tau_{1}, \tau_{2} \in \mathrm{C}([0, \infty),(0, \infty))$ with $\mathrm{t}-\tau_{\mathfrak{i}}(\mathrm{t}) \rightarrow \infty$ as $\mathrm{t} \rightarrow \infty, \mathfrak{i}=1,2$. A simple calculation shows that

$$
\begin{gathered}
g(0)=f(0)=q(t, 0,0)=0, \int_{0}^{\infty} a(s) d s=\infty, \\
L_{1}(t)=L_{2}(t)=\frac{1}{16(t+1)}, L_{g}=1, L_{f}=1, \\
\int_{0}^{t} e^{-\int_{u}^{t} a(s) d s}\left[L_{g}|b(u)|+L_{f}|c(u)|+L_{1}(u)+L_{2}(u)\right] d u \leq \frac{11}{40},
\end{gathered}
$$

and

$$
\begin{aligned}
& a(t) \int_{0}^{t} e^{-\int_{u}^{t} a(s) d s}\left[L_{g}|b(u)|+L_{f}|c(u)|+L_{1}(u)+L_{2}(u)\right] d u \\
& +L_{g}|b(t)|+L_{f}|c(t)|+L_{1}(t)+L_{2}(t) \leq \frac{22}{40} .
\end{aligned}
$$

It is easy to see that all the conditions of Theorem 2.3 hold for $\alpha=\frac{22}{40}<1$. Thus, Theorem 2.3 implies that the zero solution of (2.13) is globally asymptotically stable in $\mathrm{C}^{1}$. 
Acknowledgments. The authors would like to thank the anonymous referee for his/her valuable remarks. 


\section{References}

[1] M. Adıvar, Y. N. Raffoul, Existence of periodic solutions in totally nonlinear delay dynamic equations. Electronic Journal of Qualitative Theory of Differential Equations 2009, 1 (2009), $1-20$.

[2] A. Ardjouni, I. Derrardjia and A. Djoudi, Stability in totally nonlinear neutral differential equations with variable delay, Acta Math. Univ. Comenianae, Vol. LXXXIII, 1 (2014), pp. 119-134.

[3] A. Ardjouni, A Djoudi, Existence of periodic solutions for nonlinear neutral dynamic equations with functional delay on a time scale, Acta Univ. Palacki. Olomnc., Fac. rer. nat., Mathematica 52, 1 (2013) 5-19.

[4] A. Ardjouni, A Djoudi, Stability in neutral nonlinear dynamic equations on time scale with unbounded delay, Stud. Univ. Babeç-Bolyai Math. 57(2012), No. 4, 481-496.

[5] A. Ardjouni, A Djoudi, Fixed points and stability in linear neutral differential equations with variable delays, Nonlinear Analysis 74 (2011), 2062-2070.

[6] M. Belaid, A. Ardjouni and A. Djoudi, Stability in totally nonlinear neutral dynamic equations on time scales, International Journal of Analysis and Applications, Vol. 11, Nu. 2 (2016), 110123.

[7] M. Bohner, A. Peterson, Dynamic Equations on Time Scales, An Introduction with Applications, Birkhauser, Boston, 2001.

[8] M. Bohner, A. Peterson, Advances in Dynamic Equations on Time Scales, Birkhäuser, Boston, 2003.

[9] T. A. Burton, Liapunov functionals, fixed points and stability by Krasnoselskii's theorem, Nonlinear Stud. 9 (2001), 181-190.

[10] T. A. Burton, Stability by fixed point theory or Liapunov theory: A Comparaison, Fixed Point Theory, 4(2003), 15-32.

[11] T. A. Burton, Stability by Fixed Point Theory for Functional Differential Equations, Dover Publications, New York, 2006.

[12] I. Derrardjia, A. Ardjouni and A. Djoudi, Stability by Krasnoselskii's theorem in totally nonlinear neutral differential equations, Opuscula Math. 33(2) (2013), 255-272.

[13] S. Hilger, Ein Maßkettenkalkül mit Anwendung auf Zentrumsmannigfaltigkeiten, Ph. D. thesis, Universität Würzburg, Würzburg, 1988.

[14] E. R. Kaufmann, Y. N. Raffoul, Stability in neutral nonlinear dynamic equations on a time scale with functional delay, Dynamic Systems and Applications 16 (2007) 561-570. 
[15] G. Liu, J. Yan, Global asymptotic stability of nonlinear neutral differential equation, Commun Nonlinear Sci Numer Simulat 19 (2014) 1035-1041.

[16] D. R. Smart, Fixed point theorems, Cambridge Tracts in Mathematics, no. 66, Cambridge University Press, London-New York, 1974.

[17] R. Yazgan, C. Tunc and O. Atan, On the global asymptotic stability of solutions to neutral equations of first order, Palestine Journal of Mathematics, Vol. 6(2) (2017), 542-550. 